\title{
Agudeza visual en alumnos de medicina en una universidad privada de Puebla, México.
}

\author{
Dra. Cristina del Rocio Sánchez Hernández²; MsC. Liliana Rivadeneyra-Espinoza²; Dr. Álvaro González Pérez ${ }^{1}$
}

1. Universidad Popular Autónoma del Estado de Puebla; Facultad de Medicina. Departamento de Ciencias de la Salud. Puebla, México.

2. Universidad Popular Autónoma del Estado de Puebla; Coordinación de Investigación. Departamento de Ciencias de la Salud. Puebla, México. Correo electrónico: liliana.rivadeneyra@upaep.mx

Fecha de recibido: Enero 25 de 2016

Fecha de corrección: Febrero 8 de 2016

Fecha de aceptación: Marzo 14 de 2016

\section{Resumen}

Introducción. La agudeza visual se considera como un parámetro visual habitual que se emplea para evaluar la salud ocular y sirve para detectar alteraciones a bajo costo y reducir la ceguera prevenible o curable. Objetivo. Identificar las alteraciones de la agudeza visual normal, discapacidad moderada o severa y ceguera en los estudiantes de medicina que se encuentran a la mitad de la carrera a través de la toma de agudeza visual. Método. Se utilizó un diseño no experimental, descriptivo, observacional, transversal prospectivo. La muestra se conformó por 308 alumnos de la Universidad Popular Autónoma del Estado de Puebla de la carrera de Medicina que cumplían con los criterios de inclusión. Se aplicó un cuestionario el cual incluía antecedentes personales oftalmológicos y sintomatología oftálmica y se realizó la prueba de agudeza visual con la cartilla de snellen. Resultados. El 88.31\% tuvieron una agudeza visual normal, $11.68 \%$ de los alumnos presentó déficit de la agudeza visual de los cuales la discapacidad visual moderada se presentó en un $10.71 \%$ en el ojo derecho y en un $11.03 \%$ en el ojo izquierdo, la categoría de discapacidad severa el porcentaje presentado fue menor al 1\% en ambos ojos. Conclusión. En esta universidad la mayoría de los alumnos se encuentran visualmente normales, pero existe la necesidad de establecer programas continuos que permitan ir identificando a aquellos alumnos que han disminuido su agudeza visual para evitar que no se reduzca el aprendizaje y desarrollo de destrezas propias de la carrera de Medicina.

Palabras clave: Agudeza visual; Estudiantes de medicina; Ceguera
Visual acuity in students of medicine at a private university of Puebla, Mexico.

\begin{abstract}
Introduction. The visual acuity is considered as a parameter visual routine that is used to evaluate the ocular health and serves to detect alterations at low cost and reduce the preventable blindness or curable. Objective. Identify the changes in visual acuity normal, moderate or severe disability and blindness in the medical students who are at the half of the race through the taking of visual acuity. Method. Design was a non-experimental, descriptive, observational, prospective cross-sectional. The sample was composed of 308 students of the Universidad Popular Autónoma del Estado de Puebla of the medical career that fulfilled the inclusion criteria. A questionnaire was administered which included personal history ophthalmologic and symptomatology ophthalmic and conducted the test of visual acuity with Snellen's test. Results. The 88.31 $\%$ of the patients had a visual acuity normal, $11.68 \%$ of the students presented deficit of the visual acuity of which the visual disability moderate was presented in a $10.71 \%$ in the right eye and a $11.03 \%$ in the left eye, the category of severe disability the percentage was less than $1 \%$ in both eyes. Conclusion. In this university the majority of students are visually normal, but there is a need to establish ongoing programs that allow for identifying those students that have decreased their visual acuity to avoid that you do not reduce the learning and development of skills of the medical career.
\end{abstract}

Kew words: Visual acuity; Medical students; Blindness

\section{Introducción}

La agudeza visual se define como la capacidad del sistema de visión para percibir, detectar o identificar objetos especiales en condiciones de iluminación adecuadas. Es considerada como un parámetro visual habitual que se emplea para evaluar la habilidad visual. ${ }^{1-5}$

Una detección temprana de las alteraciones de la agudeza visual permite promover la salud visual, prevenir las enfermedades oculares y reducir la ceguera prevenible o curable. La ceguera y visión baja constituye un problema de salud pública a nivel mundial. Se calcula que alrededor de un $80 \%$ de los casos de ceguera pueden ser curados o prevenidos, siempre y cuando existan los servicios adecuados. ${ }^{6}$

Las alteraciones visuales producen consecuencias adversas en el individuo, lo cual limita el desarrollo adecuado en los niños y repercute en el rendimiento escolar. En los adultos la productividad se limita, por el deterioro funcional. En los estudiantes de medicina resulta importante conocer su agudeza visual debido a las limitantes que puede tener su desarrollo en áreas del conocimiento práctico dentro del ejercicio de su profesión.

El tamizaje es una forma de detectar costo-efectivo de una enfermedad para evitar progresiones, complicaciones e iniciar el tratamiento oportuno para disminuir consecuencias en la salud de una persona. ${ }^{7}$ La toma de la agudeza visual es el método más sencillo y de mínimo costo para determinar las alteraciones de la agudeza visual.

En la actualidad existen diferentes métodos para determinar el grado de agudeza visual u optotipos (test de Snellen, ETDRS, Wecker, Landolt, Bailey-Lovie, etc. ${ }^{8}$, de los cuales, el más conocido y utilizado por la OMS es el test de Snellen. En el cual el tamaño de la imagen es directamente proporcional al tamaño del test (conjunto de optotipos) e inversamente proporcional a la distancia del mismo. Normalmente la distancia de presentación de los optotipos es de 6 metros. El optotipo original de Snellen (presentado en 1862) presenta siete niveles diferentes de letras. Solo dispone de un optotipo en el tamaño mayor (mínima AV) incrementando progresivamente un optotipo (una letra) por línea hasta alcanzar 8 en la línea de AV 1,0. La progresión del tamaño de los optotipos es aritmética (razón = tangente ángulo $\times$ distancia) para las distancias (expresadas en pies) de 200, 100, 70, 50, 40, 30 y 20 (de menor a mayor AV), que en escala decimal correspondería a las AV de 0,05;0,1;0,3;0,4;0,5;0,6 y 1,0, respectivamente y $1,0 .^{9}$ 
La clasificación de la agudeza visual se realiza de acuerdo con las categorías generales de la OMS de visión "normal", discapacidad visual moderada $(<6 / 18$ y $>$ a $6 / 60$ o $<20 / 60$ y $<20 / 200)$, discapacidad visual severa $(<6 / 60$ y $>3 / 30$ o $<20 / 200$ y $>20 / 400)$ y ceguera $(<20 / 400)$. ${ }^{6,10,11}$

El objetivo de esta investigación fue identificar las alteraciones de la agudeza visual normal, discapacidad moderada o severa y ceguera en los estudiantes de medicina que se encuentran a la mitad de la carrera a través de la toma de agudeza visual, debido a que en estos semestres en el programa académico inicia la integración a rotaciones en hospitales para su formación clínica.

\section{Material y métodos}

En esta investigación se utilizó un diseño no experimental, descriptivo, observacional, transversal prospectivo.

La selección de la muestra fue de manera no probabilística (aleatoria), tomando en cuenta los criterios de inclusión los cuales fueron estar inscrito en la Universidad Popular Autónoma del Estado de Puebla (UPAEP) en la carrera de Medicina, cursar el $5^{\circ}$ o $6^{\circ}$ semestre de la carrera, tener una edad entre 19 y 28 años sin discriminación de sexo. Se excluyeron a alumnos de 1er, 2do, 3er, 4to, 7 mo y 8 vo semestre, menores de 19 años y mayores de 24 , que pertenecieran a la carrera de medicina.

Se consideraron a 308 de alumnos que cumplían con los criterios antes mencionados, un médico oftalmólogo aplico un cuestionario el cual incluía: antecedentes personales oftalmológicos y sintomatología oftálmica. Posteriormente se les realizó un estudio de agudeza visual con una cartilla de Snellen a 6 metros de distancia y se utilizó la clasificación modificada por la OMS, para desarrollar una estadística descriptiva.

\section{Resultados}

Cuadro 1. Clasificación de los alumnos de la facultad de medicina UPAEP de 5to y 6 to semestre por grupos de edad.

\begin{tabular}{lcc}
\hline Edad & Numero & $\%$ \\
\hline 19-20 años & 174 & 56.49 \\
21-22 años & 121 & 39.28 \\
23-24 años & 9 & 2.92 \\
25-28 años & 4 & 1.29 \\
Total & 308 & 100 \\
\hline
\end{tabular}

Se incluyeron 308 alumnos de 5to y 6to semestre de la facultad de Medicina de la Universidad Popular Autónoma del Estado de Puebla, de los cuales 175 eran mujeres y 133 hombres. 56.49\% tenían una edad entre 19 y 20 años, $39.28 \%$ de 21 a 22 años, $2.92 \%$ de 23 a 24 años y solo el $1.29 \%$ arriba de 25 años de edad. Cuadro 1.

Cuadro 2. Factores que influyen en el nivel de la agudeza visual.

\begin{tabular}{lcccccc}
\hline & \multicolumn{2}{c}{$\begin{array}{c}\text { Antecedentes } \\
\text { oftalmológicos }\end{array}$} & \multicolumn{2}{c}{$\begin{array}{c}\text { Síntomas } \\
\text { oftálmicos }\end{array}$} & Uso de lentes \\
\hline & Núm. & $\%$ & Núm. & $\%$ & Núm. & $\%$ \\
Presentes & 81 & 26.29 & 62 & 20.12 & 50 & 16.23 \\
No presentes & 227 & 73.70 & 246 & 79.87 & 258 & 83.76 \\
\hline
\end{tabular}

De acuerdo a las respuestas obtenidas sobre los antecedentes oftalmológicos, estos estuvieron presentes en un $26.29 \%$ de los alumnos encuestados, $20.12 \%$ presentaban en ese momento síntomas oftálmicos y 16.23\% del total usan o usaron lentes. Cuadro 2.

Figura 1. Antecedentes oftalmológicos en alumnos de la facultad de medicina UPAEP de 5to y 6to semestre.

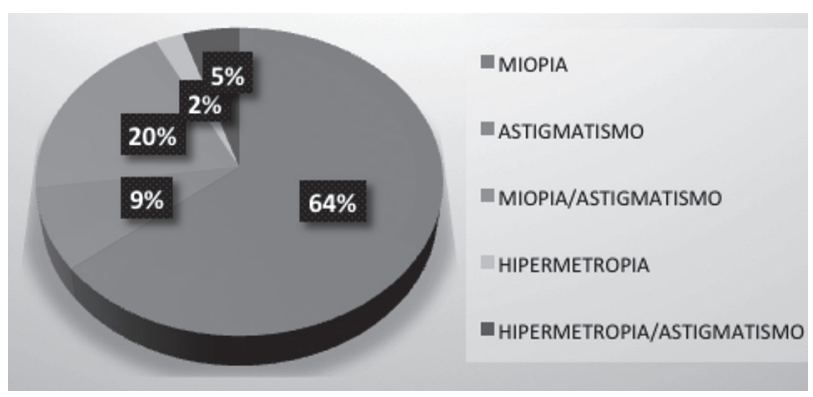

Los antecedentes oftalmológicos más frecuentemente presentes fueron miopía en un $64 \%$, en segundo lugar miopía más astigmatismo $20 \%$ y en tercer lugar astigmatismo, aunque también se presentaron hipermetropía e hipermetropía más astigmatismo. FIGURA 1.

Figura 2. Presencia de síntomas oftálmicos en alumnos de la facultad de medicina UPAEP de 5 to y 6 to semestre.

PROCENTAJE DE PRESENTACIÓN DE SINTOMAS OFTÁLMICOS

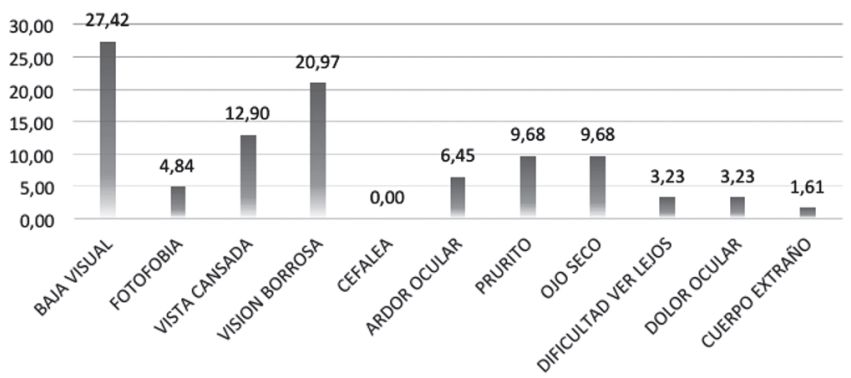

Los alumnos refirieron en un 27.42\% de los síntomas oftálmicos baja visual, en un $20.97 \%$ visión borrosa, vista cansada en un $12.90 \%$, prurito y ojo seco en un $9.68 \%, 6.45 \%$ presentaron ardor ocular, fotofobia en un $4.84 \%$, el restante refirió dificultad para ver de lejos, dolor ocular y sensación de cuerpo extraño. FIGURA 2. 
Cuadro 3. Clasificación de la Agudeza visual modificada por la OMS en los alumnos de la facultad de medicina de 5to y 6to semestre.

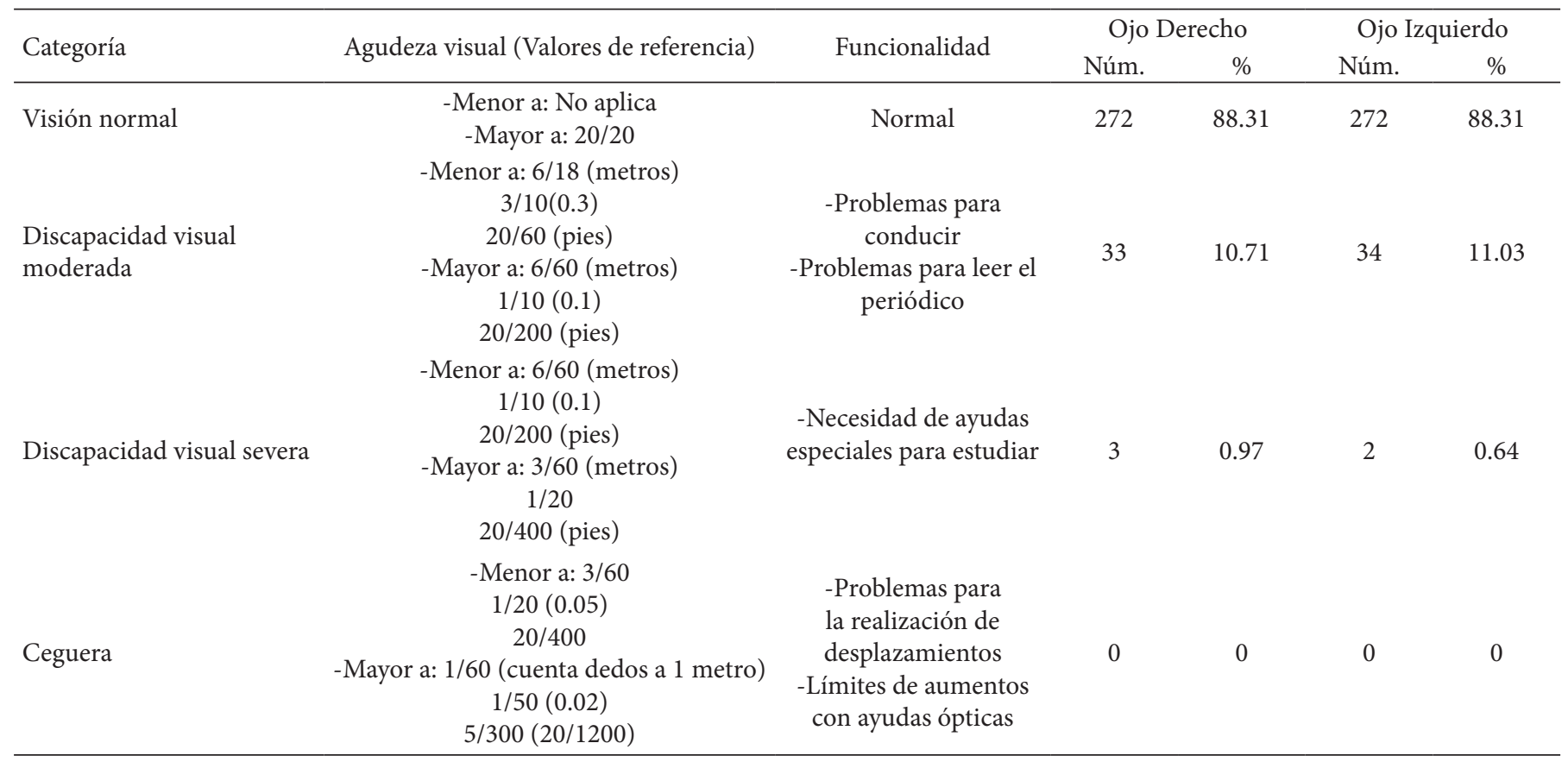

Posterior a la realización del examen con la cartilla snellen se ordenaron los resultados de acuerdo con la clasificación de la agudeza visual modificada por la OMS, el 88.31\% de los alumnos se catalogaron con una visión normal tanto en el ojo izquierdo como en el derecho, con déficit de la agudeza visual $11.68 \%$, de los cuales la discapacidad visual moderada se presentó en un $10.71 \%$ en el ojo derecho y en un $11.03 \%$ en el ojo izquierdo, por consiguiente este grupo de alumnos empiezan a tener tienen problemas de funcionalidad, como por ejemplo para conducir o leer el periódico. Por otra parte, en la categoría de discapacidad severa el porcentaje presentado fue menor al $1 \%$ en ambos ojos, aunque la funcionalidad se ve mucho más afectada, debido a que necesitan de ayuda especial para estudiar los alumnos que se encuentran en ella. En la agudeza visual menor a 20/200 o mayor a 20/1200 correspondiente a ceguera no se clasifico ningún alumno con este resultado. Cuadro 3.

\section{Discusión}

A nivel mundial, mucho se ha discutido sobre la salud visual y ocular, y según la (OMS), en la Asamblea del 2003, reconoce que la salud visual es importante para el desarrollo humano, y que todos los países deben adoptar programas de visión 20/20 y, como son muchos los problemas de salud en nuestros pueblos, puesto que influye directamente en el Índice de Desarrollo Humano (IDH), aportando la alfabetización y a la calidad de vida. ${ }^{12}$

Debido a que alrededor del $90 \%$ de las personas con visión disminuida que existen en el mundo se encuentran en países en desarrollo como México. La provisión de pruebas adecuadas para estas zonas, donde hay escasez de personal adiestrado para atender a la salud ocular y pocos recursos, resulta por lo tanto imprescindible. ${ }^{13}$ En el 2010 se estimó que vivian en las Américas aproximadamente 26.6 millones de personas con deficiencia visual, de las cuales 3.2 millones eran ciegos. ${ }^{14}$ Por eso la importancia de saber realzar un examen de agudeza visual y el impacto y/o utilidad que tienen los resultados.

La medida de la agudeza visual es un parámetro que resulta necesario para evaluar el estado de salud ocular, si bien existen enfermedades oculares que pueden afectar mínimamente a la agudeza visual o incluso no hacerlo, otras pueden causar su descenso de forma brusca o aguda. Esto cobra suma importancia ya que sirve para determinar grados de incapacidad laboral o profesional, el cual se plasma en la ley general del trabajo en el artículo 514. ${ }^{15}$

Los estudiantes de medicina y futuros médicos poseen una gran dependencia de su sistema visual para poder formarse con bases sólidas tanto teóricas como prácticas, ya que requieren de una visión adecuada para facilitar su desarrollo físico, mental, social y cultural; al ejercer su profesión de la manera más correcta.

El déficit visual se presentó en un $11.68 \%$, porcentaje muy parecido al riesgo de padecer trastornos visuales (11\%) en un estudio realizado en la Universidad Autónoma de Tlaxcala en donde se asocia este padecimiento principalmente al uso de la computadora, debido a que los universitarios desarrollan la mayoría de sus actividades académicas, personales y laborales con esta herramienta (96\%). ${ }^{16}$

Ante esta situación, se debe considerar la necesidad de que los estudiantes estén informados sobre medidas preventivas que pueden emplear para proteger su salud evitando así la presencia de este tipo de trastornos a temprana edad, ${ }^{17}$ además de saber identificar los síntomas y la referencia al presentarlos. Los alumnos con discapacidad visual moderada y severa fueron enviados a la clínica universitaria para completar el examen 
oftalmológico y tomar las medidas necesarias para su tratamiento y correcta visión.

\section{Conflicto de intereses:}

Se deja constancia que los autores no tienen conflictos de intereses y que este trabajo no ha tenido fuentes de financiamiento en ninguna de sus etapas.

\section{Agradecimientos}

A Abner Luna Hernández, Sayla Nallely Alcántara Alonso, Alfonso Rodríguez Contreras y a Jessica Alejandra Pérez Rico por el apoyo brindado en la captura de datos en esta investigación.

\section{Referencias}

1. Sánchez-Verdiguel I, Bosch V, Ordaz-Favila JC. Como identificar problemas de visión en la edad pediátrica. Acta Pediatr Mex.2011;32(4):247-250.

2. Ramos EA, Rodríguez S, Copello M, Linares M, Reselló A, Rodríguez N. Catarata congénita y baja visión. Habilitación visual en un grupo de pacientes Revista de Ciencias Médicas. 2011;10(1):61- 76

3. Sixto S, Boffill A, Jalilo SM, González DC. Evaluación de factores de riesgo en accidentes oculares graves infantiles. Rev Ciencias Médicas.2010;14(3)

4. Verrone PJ, Simi MR. Prevalencia de agudeza visual baja y trastornos oftalmológicos en niños de seis años de la ciudad de Santa Fe. Arch Argent Pediatr. 2008;106(4):328-333/328.

5. Cruz D, Castillo A. Caracterización epidemiológica del trauma ocular pediatrico a globo abierto en zona I en el Instituto Cubano de Oftalmología "Ramón Pando Ferrer". Rev Cubana Oftalmol.2011;24(1)

6. Ceguera y discapacidad visual. Organización Mundial de la Salud. 2014. Disponible en: http://www.who.int/mediacentre/factsheets/ fs $282 /$ es/

7. Rincón IA, Rodríguez NC. Tamización de salud visual en población infantil: prevención de la ambliopía. Repert.med.cir. 2009;18(4):210-17.

8. Vargas JG. Agudeza visual desde lo analítico en lentes de contacto. Rev Panamericana de lentes de contacto. 2011;3(2)

9. Dominguez-Palacios M. Correlación entre agudezas visuales de lejos y cerca en pacientes de baja visión. Gaceta óptica. 2011;407

10. International classification of impairments, disabilities, and handicaps. Geneva: World Health Organization; 1980.

11. Suárez JC. Adult visual impairment and blindness: Review article. Medicina U.P.B. 2011;30(2):170-180.

12. Arellano G, Chávez A, Arellano S, Chaves C. Estudio de la agudeza visual y problemas refractivos en estudiantes de medicina de la escuela superior politécnica de Chimborazo. SCientifica. 2014;12(1):13-19

13. Keeffe JE, Lovie-Kitchin JE,Maclean H, Taylor HR. Prueba de tamizaje simplificada para identificar a personas con visión disminuida en países en desarrollo. Rev Panam Salud Publica/Pan Am J Public Health. 1998;3(4):220-226
14. Campos B, Cerrate A, Montjoy E, Dulanto Gomero V, Gonzalez C, Tecse A, et al. Prevalencia y causas de ceguera en Perú: encuesta nacional. Rev Panam Salud Pública. 2014;36(5):283-9.

15. Estados Unidos Mexicanos. Secretaria del trabajo y previsión social. Ley federal del trabajo: artículo 514. Disponible en: http://www.stps.gob.mx/bp/micrositios/reforma_laboral/ archivos/Noviembre.\%20Ley\%20Federal\%20del\%20Trabajo\%20 Actualizada.pdf

16. Lumbreras I, Moctezuma MG, Dosamantes LD, Medina MA, Cervantes M, López MRR, et al. Estilo de vida y riesgos para la salud en estudiantes universitarios: hallazgos para la prevención. Revista digital Universitaria. 2009;10(2)

17. Tamez-González S, Ortiz L, Martínez-AlcántaraS, MéndezRamírez I. "Riesgos y daños a la salud derivados del uso de videoterminal”.Salud Pública de México. 2003;45(3):171-180. 